\title{
Journal of the Mechanical Behavior of
} Biomedical Materials

\section{Effect of primer-cement systems with different functional phosphate monomers on the adhesion of zirconia to dentin}

\author{
Dayanne Monielle Duarte Moura $^{\mathrm{a}}$, Ana Beatriz do Nascimento Januário ${ }^{\mathrm{a}}$, \\ Arthur Magno Medeiros de Araújo ${ }^{a}$, Amanda Maria de Oliveira Dal Piva ${ }^{b}$, Mutlu Özcan ${ }^{c}$, \\ Marco Antonio Bottino ${ }^{\mathrm{b}}$, Rodrigo Othávio Assunção Souza ${ }^{\mathrm{a}, *}$ \\ a Federal University of Rio Grande do Norte (UFRN), Department of Dentistry, Lagoa Nova, Natal, Brazil \\ ${ }^{\mathrm{b}}$ São Paulo State University (UNESP), Institute of Science and Technology, São José dos Campos, Brazil \\ ${ }^{\mathrm{c}}$ University of Zürich, Dental Materials Unit, Center for Dental and Oral Medicine, Clinic for Fixed and Removable Prosthodontics and Dental Materials Science, Zurich, \\ Switzerland
}

\section{A R T I C L E I N F O}

\section{Keywords:}

Adhesion

Dentin

Resin Cement

Phosphate monomer

Shear Bond Strength

Zirconia

\begin{abstract}
A B S T R A C T
Purpose: The objective of this study was to evaluate the effect of primer-cement systems with different functional phosphate monomers on the adhesion of zirconia to dentin with and without aging protocols.

Materials and methods: Bovine teeth $(\mathrm{N}=180)$ were embedded in acrylic resin after sectioning their roots with with their coronal parts exposed. The buccal surface of each tooth was polished with silicon carbide papers (\#200, 400, 600) until dentin exposure. Sintered zirconia cylinders $(\mathrm{N}=180)(\varnothing: 3.4 \mathrm{~mm}$; height: $4 \mathrm{~mm})(\mathrm{Vita}$ In-Ceram 2000) were prepared and distributed into 18 groups ( $n=10$ per group) considering the following factors: "Cementation System" (Panavia F - PAN; RelyX Ultimate - ULT, Multilink N - MULT) and "aging" (water storage in distilled water at $37^{\circ} \mathrm{C}$ for $24 \mathrm{~h}$ (control, C); 30 days (30D); 6 months $(6 \mathrm{M})$ and thermocycling for 5000 (5TC), 10,000 (10TC) and 20,000 (20TC) thermal cycles (5-55 ${ }^{\circ} \mathrm{C}$; dwell time: $\left.30 \mathrm{~s}\right)$ ”. Zirconia and dentin cementation surfaces were conditioned according to the recommendations of the manufacturers of each resin cement. The cylinders were adhesively cemented to the dentin surfaces and the specimens were submitted to the aging protocols. After aging, the specimens were subjected to shear bond strength test (SBS) (1 mm/min) in a Universal Testing Machine and failure types were analyzed. The data (MPa) were statistically using KruskalWallis followed by the Dunn test $(\alpha=5 \%)$. The degree of conversion (DC) rates of the cementing systems were also measured.

Results: While without aging $(24 \mathrm{~h})$ no significant difference was found between the cement systems ( $\mathrm{p}>0.05)$, after 30D (4.3-5.4), the highest decrease in all groups were observed after 5TC (1.5-2.3) ( $<<0.05)$. Overall, MULT and ULT presented significantly higher results than that of PAN ( $\mathrm{p}<0.05)$. Pre-test failures during TC were more frequent in the PAN group. Complete adhesive failures at the cement/dentin interface were more frequent for MULT (30-80\%) and PAN (10-70\%) and for ULT (20-90\%) at the cement/ceramic interface. DC of the tested cements did not show significant difference.

Conclusion: Adhesion performance of the primer-cement systems with different functional phosphate monomers on zirconia-dentin complex varied as a function of aging strategies with MULT and ULT delivering higher bond strength values. When failure types considered, none of the cement systems performed well on both ceramic and dentin.
\end{abstract}

\section{Introduction}

During the last two decades, there has been an increase in the use of zirconium-oxide-based ceramic (hereon: zirconia) for the fabrication of metal-free restorations due to its high flexural strength
(900-1200 MPa), resistance to chemical corrosion, aesthetics, biocompatibility and low bacterial adhesion (Denry and Kelly, 2008; Egilmez et al., 2013). From clinical perspective, zirconia could be considered as a versatile material with several possibilities of clinical applicability such as customized abutments (Bachhav and Aras, 2011;

\footnotetext{
* Correspondence to: Federal University of Rio Grande do Norte (UFRN), Department of Dentistry, Av. Salgado Filho, 1787, Lagoa Nova, Natal CEP: 59056-000, RN, Brazil.

E-mail address: rodrigoothavio@dod.ufrn.br (R.O.A. Souza).
} 
Neiva et al., 1998), fixed dental prosthesis (FDP) (Tinschert et al., 2001), frameworks of single crowns or multiple unit FDPs or monolithic crowns (Stober et al., 2014). With the advances with ultra-translucent zirconia ceramics, even ultrathin monolithic veneers could be fabricated (Souza et al., 2018).

Clinical studies reported high survival rates of zirconia when used as framework of single crowns $(88.8 \%$ and $98.3 \%$ at 3 and 5 years, respectively) (Ortorp et al., 2012) and FDPs (96.3\% up to 3 years) (Schmitt et al., 2009). However, despite the high survival rates, several failures have been reported in the literature, such as veneer ceramic fracture/chipping, marginal discoloration, fracture of the framework and debonding of the FDPs (Schley et al., 2010; Solá-Ruíz et al., 2015). Many studies have reported that the debonding is still one of the most common failures in zirconia FDPs (Ohlmann et al., 2008) due to little or no mechanical retention of resin materials on highly crystalline structure of the ceramic regardless of the cementation protocol employed (Cavalcanti et al., 2009; Chai et al., 2010; Özcan and Bernasconi, 2015).

While hydrofluoric acid and silanization creates perfect adhesion to glassy matrix ceramics, this conditioning protocol is not effective for zirconia due to the lack of glass phase (Chai et al., 2010). For this reason, several surface conditioning methods have been suggested based on mechanical or chemical conditioning principles (Yun et al., 2010; Özcan and Bernasconi, 2015) such as air-borne particle abrasion using aluminum oxide particles $\left(\mathrm{Al}_{2} \mathrm{O}_{3}\right)$, silicatization, laser (Nd: YAG, Er: YAG), selective infiltration-etching (SIE), application of low-fused porcelain, plasma spray and the use of 10-Methacryloxydecyl Dihydrogen Phosphate monomer (MDP) based primers and cements (Derand et al., 2005; Cavalcanti et al., 2009; May et al., 2010; Bottino et al., 2014). On the other hand, metal primers increase the adhesion of resin cements to zirconia through chemical reaction. The most commonly used metal primers for zirconia ceramics are based on 6-[4-vinylbenzyln-propylamino-1,3,5-triazine-2,4-dithione (VBATDT), thiophosphoric methacrylate (MEPS), 6-methacryloyloxyhexyl-2-thiouracil- 5-carboxylate (MTU-6), MDP alone or in combination of othe functional monomers (Pereira et al., 2015). MDP was first marketed by Kuraray Medical Inc (Okayama, Japan) and it is currently present either in conventional or universal (multi-mode) adhesive resins, resin cements and metal primers. MDP is a monomer derived from the reaction of methacrylic acid with phosphoric acid or carboxylic acid that increases the adhesion of resin materials to zirconia through chemical bonds $(\mathrm{P}=\mathrm{O}, \mathrm{OH}=\mathrm{Zr}$ ) or ionic bonds (Perdigão and Loguercio, 2014; Kim et al., 2015; Nagaoka et al., 2017).

Currently available resin cement systems vary in chemical compositions, physical properties, modes of activation and interaction with dentin where the latter could be chemically-, photo- or dual-polymerized, or could be simply self-adhesive, and may or may not contain MDP in their composition (Tanis et al., 2015). As for conditioning the tooth substance, the so-called universal adhesive systems on the other hand, could be based on etch-and-rinse (ER) or self-etch (SE) adhesives on dentin and on ER adhesive systems on enamel. Universal adhesives, such as Scotch Bond Universal (SBU), on the other hand act as a primer for metal and zirconia due to the MDP in its composition. SBU also contains Vitrebond copolymer, water, and silane, which make it a versatile material that can be used for different substrates including resin composites, glass ceramics, zirconia, and metallic alloys, making it an efficient agent for achieving adhesion at the ceramic-cementdentin interface (Kim et al., 2015).

The clinical longevity of zirconia-based ceramic reconstructions also depends on the cement -dentin interface, especially in situations where the mechanical retention of the preparation is not ideal (Bottino et al., 2014). Adhesion to dentin is still a challenge due to the different interfaces involved, substrate-adhesive system- cement interactions. In addition, zirconia-cement-dentin interface makes overall adhesion of such reconstructions more challenging. Moreover, these interfaces are constantly subjected to mechanical, chemical and thermal stresses and may react to such aging conditions in different ways, affecting longevity of the reconstructions. Thus, using a cementation strategy that promotes a durable adhesion of the resin cement to both tooth surface and zirconia is important to ensure the success of the restoration (Alves et al., 2016). Some studies have evaluated the adhesion of zirconiabased ceramics involving dentin and reported that conditioning methods used for tooth and zirconia and the type of resin cement used may affect the adhesion of zirconia (Chai et al., 2011; Bottino et al., 2014; Alves et al., 2016). However, limited information is available on the adhesion of zirconia ceramics to dentin using universal adhesive systems (Alves et al., 2016).

From the clinical point of view, durability of adhesion depends not only on the interaction between zirconia- cement-dentin complex but also on the micromechanical and chemical surface conditioning system, the composition of the resin cement systems, along with their resistance to moisture, thermal or mechanical stresses in the oral environment (Özcan and Bernasconi, 2015; Tsujimoto et al., 2017). According to Chen et al. (2017), water plays a fundamental role in the reduction of adhesion either through a faster hydrolytic degradation of the interface or stresses due to contraction and expansion as a consequence of different thermal expansion coefficients between the tooth substrates and restorative materials. The fatigue process in particular is promoted by artificial aging and thermocycling which is considered as an important predictor of the adhesion performance of a material to a given substrate (Özcan and Bernasconi, 2015).

The objectives of this study therefore were to evaluate the effect of primer-cement systems with different functional phosphate monomers on the adhesion of zirconia to dentin with and without aging protocols. The hypotheses tested were that 1) the type of primer and cement system would affect the adhesion of zirconia to dentin, 2) aging would decrease the adhesion at the zirconia-cement-dentin complex and 3) the degree of conversion of resin cements with MDP monomers would show difference.

\section{Material and methods}

The brands, manufacturers, chemical compositions and batch numbers of the materials used in this study and the cementation protocol for ceramic and dentin surfaces are listed in Table 1 .

\subsection{Tooth specimen preparation}

Sound bovine incisors $(\mathrm{N}=180)$ were selected and cleaned with periodontal curettes until total removal of the periodontal ligament and stored in distilled water at $4{ }^{\circ} \mathrm{C}$ (ISO 11405, 2003) until the experiments. The roots were sectioned at the cement-enamel junction with a diamond disk (KG Sorensen, Cotia, São Paulo, Brazil) under constant irrigation. The crowns were embedded in chemically activated acrylic resin (JET Clássico, Campo Limpo, São Paulo, Brazil) with the buccal surfaces exposed. The buccal surfaces were subsequently flattened so that the surfaces were as parallel as possible to the acrylic resin base. Next, the dentin surface was polished in a polishing machine (Politriz, Arotec, Cotia, São Paulo, Brazil) under constant irrigation using silicon carbide papers in the decreasing grain order of \# 200, \# 400 and \# 600 (3 M ESPE/USA) for $60 \mathrm{~s}$ each in order to expose approximately 5-6 mm diameter of deep dentin, simulating the clinical conditions where an FDP is bonded on dentin. The teeth included in the study were kept in distilled water at all times in order to avoid dehydration prior to adhesion procedures.

\subsection{Ceramic specimen preparation}

Zirconia ( $=4$ ) (Vita In-Ceram-YZ2000, Bad Säckingen, Germany) $\left(20 \times 19 \times 15.5 \mathrm{~mm}^{3}\right)$ blocks were sectioned using a cutting machine (Isomet 1000, Precision Sectioning Saw, Buehler, Lake Bluff, Illinois, USA) at low speed (200 rpm) under water cooling in order to produce smaller specimens $\left(20 \times 15 \times 5 \mathrm{~mm}^{3}\right)$. The surfaces were polished using 


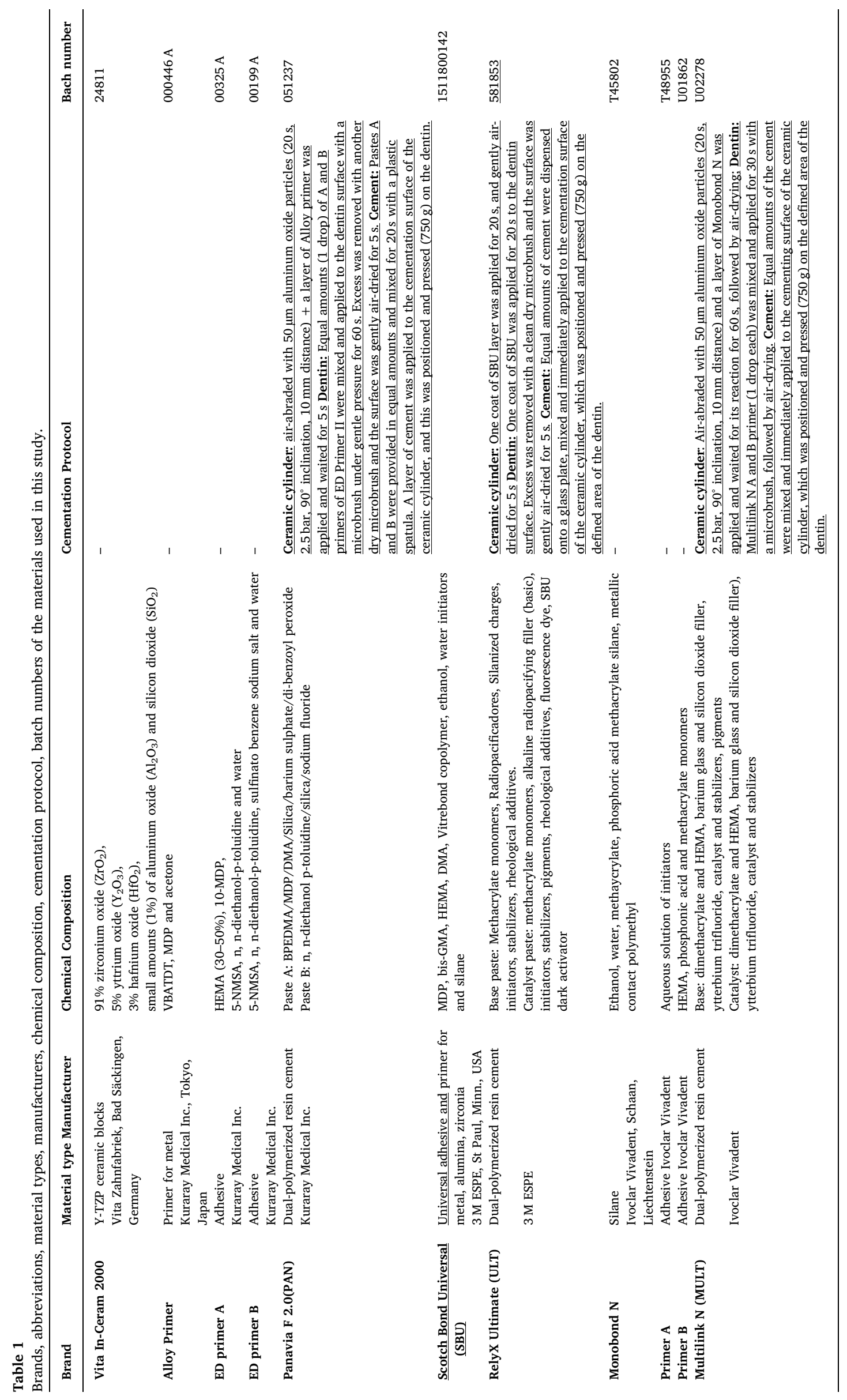




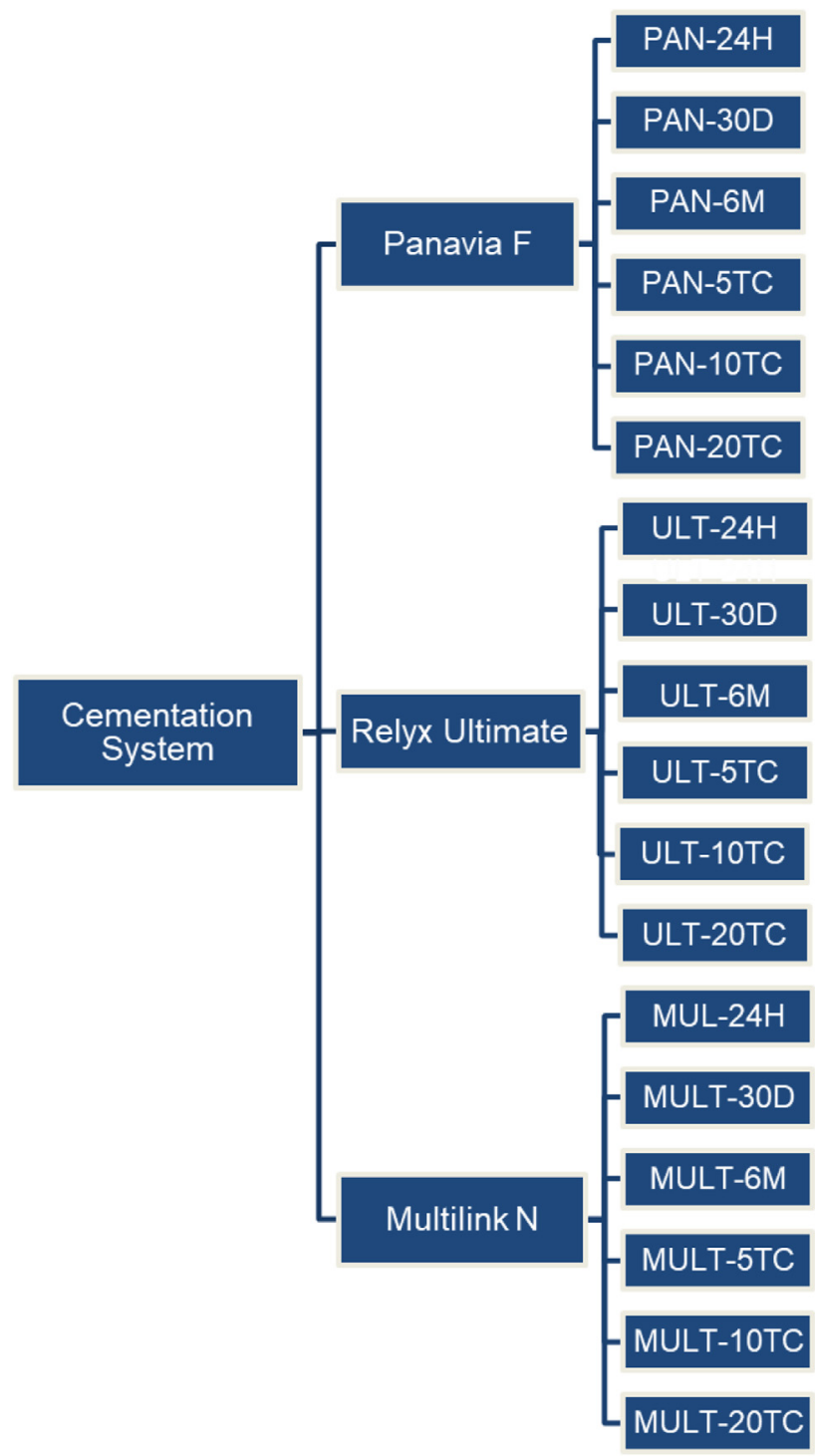

Fig. 1. Flowchart of the allocation of experimental groups ( $\mathrm{n}=10$ per group) with 2 experimental factors based on "Cementation system" -3 levels and "Aging" - 6 levels. PAN: Panavia F 2.0; ULT: RelyX Ultimate; MULT: Multilink N. H: Hours; D: Days; M: Months; TC: Thermocycling.

silicon carbide papers with grain size of \#600 and \#1200 in sequence for $20 \mathrm{~s}$ each to achieve smooth and even surfaces. A trephine bur was used to fabricate cylinders of $4.5 \mathrm{~mm}$ diameter and $5 \mathrm{~mm}$ height. The cylinders were subsequently subjected to sonic cleaning for $360 \mathrm{~s}$ in isopropyl alcohol and then submitted to sintering in a specific furnace (VITA Zyrcomat, Vita Zanhfabrik). Considering that the sintering contraction of the ceramic is approximately $20 \%$, after sintering cylinders presented final dimensions of $3.4 \mathrm{~mm}$ in diameter and $4 \mathrm{~mm}$ in height, verified with the aid of a digital caliper (Eccofer, São Paulo, Brazil). After sintering, the zirconia specimens were cleaned in an ultrasonic bath (Cristófoli, Paraná, Brazil) with isopropyl alcohol for $5 \mathrm{~min}$.

The teeth were then randomly divided into 18 groups according to the following factors: "Cementation System" (Panavia F - PAN; RelyX Ultimate - ULT, Multilink N - MULT) (3 levels) and "aging" (water storage in distilled water at $37^{\circ} \mathrm{C}$ for $24 \mathrm{~h}$ (control), C); 30 days (30D); 6 months $(6 \mathrm{M})$ and thermocycling for 5000 (5TC), 10,000 (10TC) and 20,000 (20TC) thermal cycles $\left(5-55^{\circ} \mathrm{C}\right.$; dwell time: $\left.30 \mathrm{~s}\right)$ " (6 levels) (Fig. 1).

\subsection{Surface conditioning of substrates and bonding}

Zirconia and dentin cementation surfaces were conditioned according to the recommendations of the manufacturers of each resin cement.

The zirconia specimens that were cemented with PAN and MULT resin cements had their cementation surfaces air abraded with $50 \mu \mathrm{m}$ aluminum oxide particles $\left(20 \mathrm{~s}, 2.5 \mathrm{bar}, 90^{\circ}\right.$ inclination, $10 \mathrm{~mm}$ distance) using an adapted micro-abrasion device (Microart Standard Bioart, São Carlos, SP, Brazil). Next, the surfaces were cleaned ultrasonically in distilled water for $2 \mathrm{~min}$ and air-dried. On the other hand, the zirconia specimens cemented with ULT cement had their cementation surfaces treated with SBU, as suggested by previous studies (Amaral et al., 2014; Pereira et al., 2015; Alves et al., 2016).

Prior to cementation procedures, the dentin surfaces were cleaned with a rotating brush and pumice stone in low rotation and washed with an air jet and water for $30 \mathrm{~s}$. The excess moisture was removed with absorbent paper and then the adhesive area was defined by positioning an adhesive tape (Scotch, $3 \mathrm{M}$, Ribeirão Preto, SP, Brazil) with an opening of $4.5 \mathrm{~mm}$ diameter. The cementation protocols for each cement are described in Table 1 . The zirconia specimens were placed on top of the bonding area defined by the adhesive tape and a load of $750 \mathrm{~g}$ was applied on the cylinders for $60 \mathrm{~s}$. While load was applied, excess resin cement was removed with a microbrush and the interface was photo-activated using an LED photo-polymerization device $(1200 \mathrm{~mW} /$ $\mathrm{cm}^{2}$ ) (Radii Cal, SDI, Bayswater, Victoria, Australia) for $20 \mathrm{~s}$ from four directions. After removal of the load, photo-polymerization was repeated for another $20 \mathrm{~s}$ in the same manner. The adhesive tape was then removed and the specimens were subjected to aging conditions.

\subsection{Aging of the specimens}

After cementation, the specimens were submitted to aging protocols according to their experimental group: water storage in distilled water at $37^{\circ} \mathrm{C}$ for $24 \mathrm{~h}$ (control, C); 30 days (30D); 6 months (6 M) and thermocycling for 5000 (5TC), 10,000 (10TC) and 20,000 (20TC) thermal cycles $\left(5-55^{\circ} \mathrm{C}\right.$; dwell time: $30 \mathrm{~s}$; transition time from one bath to the other: $2 \mathrm{~s}$ ).

\subsection{Shear bond strength testing}

A metal jig was used to position the specimens on the testing machine so that the ceramic-cement-dentin interface was perpendicular to the horizontal plane. A chisel shaped device (Odeme Biotechnology, Luzerna, SC, Brasil) coupled to the Universal Testing Machine (Shimadzu, AGS-X, Kyoto, Japan) with a load cell of $100 \mathrm{Kgf}$ applied the load onto the interface at a constant speed of $1 \mathrm{~mm} / \mathrm{min}$ until debonding. The bond strength calculation was performed using the formula: $\mathrm{R}=\mathrm{F} / \mathrm{A}$, where $\mathrm{R}=$ adhesive strength/shear bond strength (MPa); $\mathrm{F}=$ force $(\mathrm{N}) ; \mathrm{A}=$ interfacial area $\left(\mathrm{mm}^{2}\right)$. The adhesive area of each ceramic block defined by the area of the circle and calculated using the following formula: $A=\pi r^{2}$, where $\pi=3.14$ and $\mathrm{r}=1.7 \mathrm{~mm}$ (half of the cylinder's diameter). According to this formula, the cross-sectional area was $9.07 \mathrm{~mm}^{2}$.

\subsection{Failure analysis}

The surfaces of the debonded specimens were examined using an optical stereomicroscope (Nikon SMZ 800, New York, USA) and the representative failure types were analyzed in Scanning Electron Microscope (SEM) at x20, x40 and x70 magnification (Hitachi TM 3000, Tokyo, Japan). Failure modes were classified as follows: $\mathrm{A} 1=$ Adhesive failure at cement-dentin interface with no resin remnants on dentin; A2 = Adhesive failure at cement-ceramic interface with no resin remnants on ceramic; $\mathrm{C} 1=$ Cohesive in the cement with some remnants left on dentin; $\mathrm{C} 2=$ Cohesive failure in the cement with 
some remnants left on ceramic.

\subsection{Degree of conversion}

Sixty new specimens were prepared according to the following groups $(n=10)$ : Panavia (PAN), Panavia + ED primer (PAN + ED), RelyX Ultimate (ULT), RelyX Ultimate + Scotch Bond Universal $(\mathrm{ULT}+\mathrm{SBU})$, Multilink N (MULT), and Multilink N + AB (MULT+ Primer A and B). Cementation systems were tested with and without the use of an adhesive system.

In groups tested with adhesive system, the adhesive system was applied on a polyester strip placed on a glass plate and then equal parts of its respective resin cement were manipulated and applied on the adhesive. Next, the cement was covered with another polyester strip, a zirconia cylinder identical to that used for the shear test was positioned on the strip and pressure was applied $(750 \mathrm{~g})$ simulating a clinical procedure. Each side was photo-polymerized using a LED photo-polymerization device (Radii Cal) for $20 \mathrm{~s}$. In the other groups, without adhesive system, the specimens were made as described above, but without applying the adhesive system.

All specimens were stored for $24 \mathrm{~h}$ in black opaque Eppendorf tubes in order to prevent light exposure. Afterwards, specimens were submitted to degree of conversion analysis (DC) by measuring the maximum absorption peak at 1638 and $1608 \mathrm{~cm}^{-1}$. These values were used in the equation:

$\mathrm{DC}=\left[1-\left(\frac{\operatorname{Rpolym}\left(\frac{1638}{1608}\right)}{\operatorname{Rnpolym}\left(\frac{1638}{1608}\right)}\right)\right] x 100$

where Rpolym is the polymerized material and Rnpolym the non-polymerized material. DC was measured using Fourier Transform Infrared Spectroscopy - FTIR (Spectrum 65, PerkinElmer, São Paulo, SP, Brazil).

\subsection{Statistical analysis}

The number of debondings during thermocycling were considered as $0 \mathrm{MPa}$ in the statistical analysis (Barbosa et al., 2013). Data were analyzed using a statistical software package (Statistix 9.1 software, Chicago, IL, USA). Kolmogorov-Smirnov and Shapiro-Wilk tests were used to test normal distribution of the data. Values of mean, standard deviation, maximum and minimum were calculated. The non-parametric Kruskal-Wallis test was used due to the non-normality of the data, followed by the Dunn test (5\%). DC data were analyzed using twoway ANOVA and Tukey's post-test (5\%). P values less than 0.05 were considered to be statistically significant in all tests.

\section{Results}

\subsection{Shear bond strength}

Shear bond strength values (median \pm interquartile range) (MPa) of the three cements studied as a function of aging parameters are presented in Fig. 2. Both the "cementation systems" $(p=0.000)$ and "aging" ( $\mathrm{p}=0.000$ ) parameters significantly affected the bond strength results. Overall, PAN (2.57) resin cement presented significantly less $(\mathrm{p}<0.05)$ median value than those of ULT (5.30) and MULT (8.00) cements, where the latter two were not significant $(p>0.05)$.

Regardless of the cement type, water storage aging for the period 30D (8.8) showed slightly less aging effect yet being not significantly different compared to other water storage groups: $24 \mathrm{~h}(6.32)$ and $6 \mathrm{M}$ (6.87) ( $p>0.05)$. Aging through thermocycling significantly decreased ( $\mathrm{p}<0.05$ ) the bond strength when compared to all water storage groups (5.000: 4.04; 10.000: 1.99; 20.000: 3.73) (Table 2). The increased number of cycles above 5.000 did not significantly affect the results $(\mathrm{p}>0.05)$.
While without aging ( $24 \mathrm{~h})$ no significant difference was found between the cement systems ( $p>0.05$ ), after 30D (4.3-5.4), the highest decrease in all groups were observed after 5TC $(1.5-2.3)(\mathrm{p}<0.05)$. Overall, MULT and ULT presented significantly higher results than that of PAN ( $\mathrm{p}<0.05)$.

\subsection{Failure analysis}

The groups with the greatest number of pre-test failures during aging were PAN-10TC and PAN-20TC (50\%), followed by PAN-5TC (40\%) (Table 3).

Partial cohesive failures on the cement-dentin interface (Score C1) was the highest for ULT (10-80\%) compared to PAN and MULT (0\%) and the cement-ceramic interface (Score C2) for PAN (30-100\%) and MULT (20-70\%) compared to ULT (0\%) (Fig. 2a-b). Complete adhesive failures at the cement-dentin interface were more frequent for MULT (30-80\%) (Score A1) and PAN (10-70\%) and for ULT (20-90\%) at the cement-ceramic interface (Score A2) (Fig. 3a-b and Fig. 4 a-b)

\subsection{Degree of conversion}

Dc values did not show significant difference for cement type ( $\mathrm{p}=0.50)$ and adhesive system $(\mathrm{p}=0.41)$ but the interaction between cement and adhesive system was significant $(\mathrm{p}=0.01)$. The MULT group (68.3\%) presented the highest DC values and MULT + ED (44.7\%) the lowest but there were no significant difference between the experimental groups (Table 4).

\section{Discussion}

This study was undertaken in order to investigate the effect of primer-cement systems with different functional phosphate monomers on the adhesion of zirconia to dentin with and without aging protocols. Based on the results of this study, since the primer and cement system and aging showed a significant effect on the adhesion of zirconia to dentin the first and second hypothesis could be accepted. The DC did not show significant differences between the materials tested. Thus, the third hypothesis could be rejected.

In previous studies, the bond strength between zirconia-cementdentin complex has been tested through different tests such as macrotensile, micro-tensile, macro-shear and micro-shear (Dagostin and Ferrari, 2002; Aboushelib et al., 2007; Miragaya et al., 2011; Yang et al., 2010). Although widely used in bond strength testing, microtensile test requires cutting procedures to perform the test, which is not feasible due to the high strength and hardness of zirconia-based ceramics as it may induce premature failure at the zirconia-cement interface prior to testing (Cavalcanti et al., 2009; Passia et al., 2016). On the other hand, since shear bond strength is a simple low-cost protocol and does not require sectioning procedures (Tanis et al., 2015; Alves et al., 2016) this test method was chosen in this study. With shear tests, distribution of tensile stresses at the adhesive interface is not the same as in microtensile and may cause cohesive failure of the substrates (Kim et al., 2014) but in this study no cohesive failures were experienced neither in dentin nor in zirconia. This is most likely due to less favourable adhesion values obtained for zirconia-cement-dentin interface.

In this study, bovine dentin substrate was used as it has similar anatomical, histochemical, and radiodensity characteristics and similar adhesion values compared to human dentin, in addition to the difficulty in obtaining human teeth. Deep bovine dentin, as used in this study, generally presents a lower density of dentinal tubules, which promotes inferior adhesion compared to superficial dentin. However, we chose to work with the deep dentin in order to simulate the clinical situation where FDPs are bonded to deep dentin (Nakamichi et al., 1983; May et al., 2010; Soares et al., 2016).

Based on the results, the hypothesis that type of primer/cement 


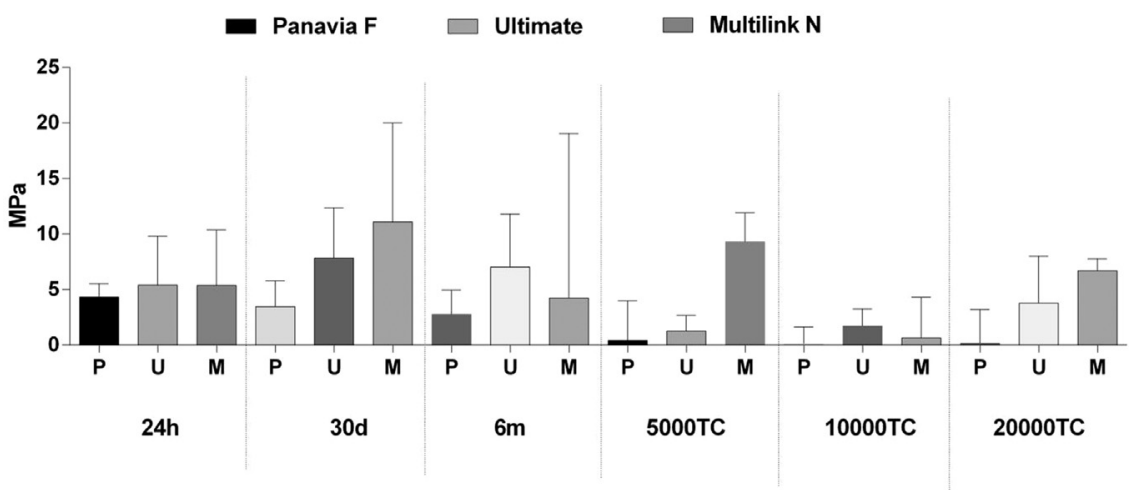

Fig. 2. Median \pm interquartile range of values obtained from the shear bond test (MPa) for the three cement systems at each aging period.

system influences the bond strength of the zirconia/cement /dentin complex was accepted. Considering the cement factor alone, ULT/SBU and MULT/ Monobond N were similar to each other and higher than that of PAN/Alloy primer combination. The three primer/cement systems evaluated in this study were selected because they are indicated for adhesive cementation of zirconia ceramic restorations as they contain MDP functional monomers. Several studies reported that the use of primers and MDP-based cements promote a more efficient chemical bond with zirconia surfaces (Faria-e-Silva et al., 2010; Özcan and Bernasconi, 2015; Elsaka, 2016).

PAN is a resin cement that presents the MDP monomer in all its components (metallic primer, adhesive system and cement) and is the most commonly advised cement system for zirconia reconstructions (Özcan and Bernasconi, 2015). However, it has been reported that the presence of MDP in all components of the system, can lead to monomer saturation, decreasing its chemical bond to ceramic oxides and thereby less adhesion results (Munoz et al., 2013). One other study also reporting lower adhesion results with PAN attributed the reason to its being high viscosity that does not allow its penetration in the microretentions created after air-abrasion (Barbosa et al., 2013).

The second cementation strategy with ULT is in fact based on methacrylate monomers but its corresponding primer SBU, contains MDP which in turn yields to chemical bond to zircoia (Perdigao et al., 2012). According to the manufacturer's recommendations, it could be used on a variety of dental substrates based on methacrylate, zirconia or metal alloys (Kim et al., 2015). In our study, the SBU was applied on the zirconia surface without previous $\mathrm{Al}_{2} \mathrm{O}_{3}$ air-abrasion. Previous studies (Alves et al., 2016; Amaral et al., 2014, Pereira et al., 2015) have reported that on zirconia, the SBU can be used alone, without additional surface treatments, as it contains the phosphate monomer (10-MDP) in its chemical composition, which interacts chemically with zirconia increasing the adhesion. In the present study, this primer/cement system did not significantly increase bond strength values. Similar results were also found in studies comparing these systems in terms of adhesion to zirconia, where the authors concluded that even after 150 days of aging, PAN and ULT cements did not differ statistically, and that this storage period was reported to be enough to age the interfaces when compared to 3-day aging (Passia et al., 2015). They further reported that the decrease in bond strength after aging is due to the various constituents present in the chemical composition of the SBU, which might have hindered MDP chemical bonds to the zirconia (Passia et al., 2015). Inferior adhesion of SBU to zirconia can be a result of the presence of several constituents within the same tube which mutually compete upon contact with the zirconia surface, preventing the actual functional monomers (10-MDP, silane) from interacting effectively (Perdigao et al., 2012; Inokoshi et al., 2014). It also has to be noted that the pre-test failures during aging were considered as $0 \mathrm{MPa}$ in this study and therefore the results were statistically not homogenous and a nonparametric test had to be conducted for the statistical analysis. Thus, the median values were considered as bon values and they cannot be directly compared to studies where parametrtic tests were employed or pre-tests were completely eliminated from statistics.

The third and final cementation strategy used in the present study was MULT, which is similar to ULT and does not present MDP in its composition. MULT is used with the metal primer Monobond N, composed of three different functional mnomers, namely silane methacrylate, phosphoric methacrylate and sulphide methacrylate. In our study, Multilink N presented significantly better bond values than those of the other systems for MULT30D and MULT5TC groups. Corroborating our study, better bond values have been found with the use of MULT in cementation of zirconia ceramics (Azimian et al., 2012). The authors report that the three adhesive primer components (a silane component, a sulphide-containing adhesion promoter and a phosphate-containing adhesion promoter) do not negatively influence the durability of the system (Azimian et al., 2012). Moreover, the results of our study confirm the clinical evidence that the presence of phosphate monomers associated with air-abrasion procedures can provide more stable adhesion of resin cements to zirconia in oral conditions (Attia and Kern, 2011; Cebe et al., 2015).

Two aging methods were used in this study: water storage ( $24 \mathrm{~h}, 30$ days and 6 months) and thermocycling (5, 10 and 20.000 cycles). Both techniques are valuable methods for assessing the hydrolysis at the cement-substrate interface (Perdigão and Loguercio, 2014). In this study, the three cements in the stored groups showed higher initial values, which decreased significantly after thermocycling. Hydrolytic degradation effect of water at the cement-ceramic and/or dentin-cement interface is also related to the differences between the coefficient of thermal expansion between the different substrates (ceramic,

Table 2

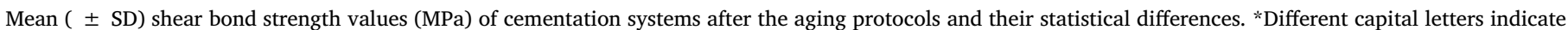
significant differences in one column and lowercase letters in one row. For group abbreviations, see Table 1.

\begin{tabular}{|c|c|c|c|c|c|c|}
\hline \multirow[b]{2}{*}{ Cement } & \multicolumn{6}{|l|}{ Aging } \\
\hline & $24 \mathrm{~h}$ & 30 days & 6 months & $5.000 \mathrm{TC}$ & $10.000 \mathrm{TC}$ & $20.000 \mathrm{TC}$ \\
\hline PAN & $4.3( \pm 1.5)^{\mathrm{Aa}}$ & $3.5( \pm 3.3)^{\mathrm{ABb}}$ & $2.8( \pm 1.7)^{\mathrm{ABa}}$ & $3( \pm 2.4)^{\mathrm{ABb}}$ & $1.7( \pm 1.3)^{\mathrm{Ba}}$ & $3.3( \pm 1.6)^{\mathrm{ABa}}$ \\
\hline ULT & $5.4( \pm 3.8)^{\mathrm{Aa}}$ & $7.9( \pm 7.2)^{\mathrm{ABab}}$ & $8.1( \pm 5.6)^{\mathrm{ABa}}$ & $1.6( \pm 1.9)^{\mathrm{ABb}}$ & $2.3( \pm 1.5)^{\mathrm{Ba}}$ & $6.4( \pm 4.3)^{\mathrm{Ba}}$ \\
\hline MULT & $5.4( \pm 9.6)^{\mathrm{ABa}}$ & $11.1( \pm 9)^{\mathrm{Aa}}$ & $4.2( \pm 9)^{\mathrm{ABa}}$ & $10.1( \pm 5.5)^{\mathrm{ABa}}$ & $1.5(5.3)^{\mathrm{Ba}}$ & $6.6( \pm 3.5)^{\mathrm{ABa}}$ \\
\hline
\end{tabular}


Table 3

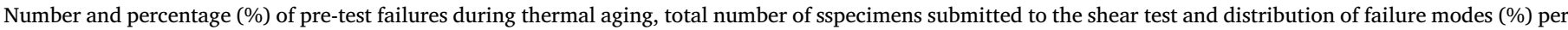

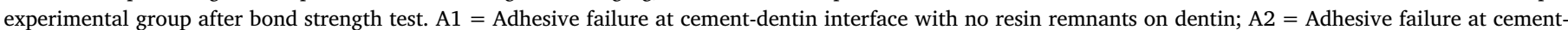

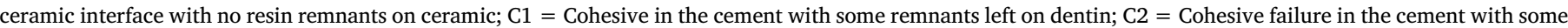
remnants left on ceramic. For group abbreviations, see Table 1.

\begin{tabular}{|c|c|c|c|c|c|c|c|c|}
\hline \multirow[t]{2}{*}{ Groups } & \multirow{2}{*}{$\begin{array}{l}\text { Number of } \\
\text { specimens }\end{array}$} & \multirow{2}{*}{$\begin{array}{l}\text { Number (\%) of spontaneous pre-test failure during } \\
\text { aging }\end{array}$} & \multirow{2}{*}{$\begin{array}{l}\text { Number (\%) of tested } \\
\text { specimens }\end{array}$} & \multicolumn{5}{|c|}{ Distribution of failure modes (\%) } \\
\hline & & & & A1 & A2 & C1 & $\mathrm{C} 2$ & Total \\
\hline PAN-24H & 10 & $0(0)$ & $10(100)$ & 60 & - & - & 40 & 100 \\
\hline PAN-30D & 10 & $0(0)$ & $10(100)$ & 50 & - & - & 50 & 100 \\
\hline PAN-6 M & 10 & $0(0)$ & $10(100)$ & 50 & - & - & 50 & 100 \\
\hline PAN-5TC & 10 & $4(40)$ & $6(60)$ & 10 & - & - & 90 & 100 \\
\hline PAN-10TC & 10 & $5(50)$ & $5(50)$ & 70 & - & - & 30 & 100 \\
\hline PAN-20TC & 10 & $5(50)$ & $5(50)$ & - & - & & 100 & 100 \\
\hline ULT-24C & 10 & $0(0)$ & $10(100)$ & - & 50 & 50 & - & 100 \\
\hline ULT-30D & 10 & $0(0)$ & $10(100)$ & - & 20 & 80 & - & 100 \\
\hline ULT-6 M & 10 & $1(10)$ & $9(90)$ & - & 50 & 50 & - & 100 \\
\hline ULT-5TC & 10 & $1(10)$ & $9(90)$ & - & 60 & 40 & - & 100 \\
\hline ULT-10TC & 10 & $2(20)$ & $8(80)$ & - & 90 & 10 & - & 100 \\
\hline ULT-20TC & 10 & $3(30)$ & $7(70)$ & - & 70 & 30 & - & 100 \\
\hline MUL-TC & 10 & $0(0)$ & $10(100)$ & 30 & - & - & 70 & 100 \\
\hline MULT-‘30D & 10 & $0(0)$ & $10(100)$ & 30 & - & - & 70 & 100 \\
\hline MULT-6 M & 10 & $0(0)$ & $10(100)$ & 80 & - & - & 20 & 100 \\
\hline MULT-5TC & 10 & $0(0)$ & $10(100)$ & 40 & - & - & 60 & 100 \\
\hline MULT-10TC & 10 & $1(10)$ & $9(90)$ & 30 & - & - & 70 & 100 \\
\hline MULT-20TC & 10 & $2(20)$ & $8(80)$ & 30 & - & - & 70 & 100 \\
\hline
\end{tabular}
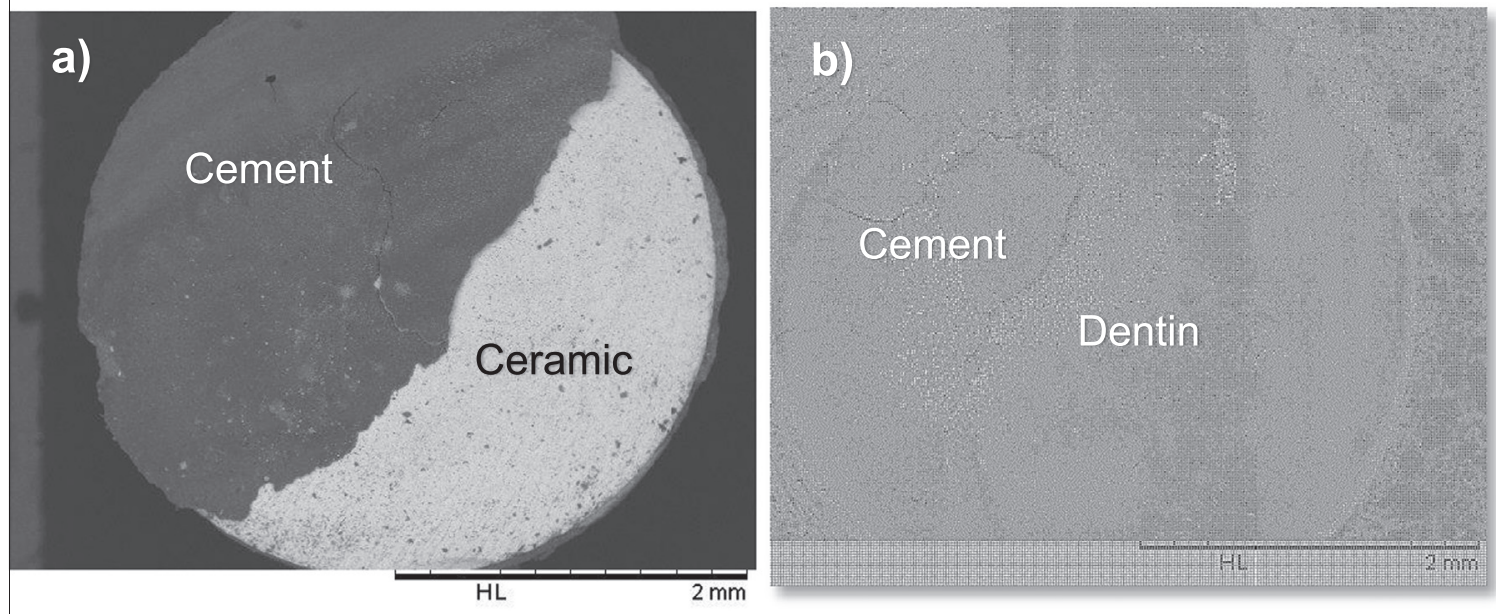

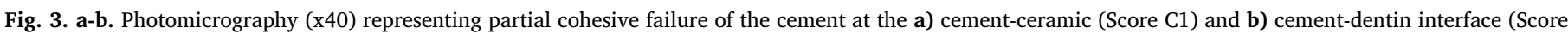
C2) for the resin cement PAN.
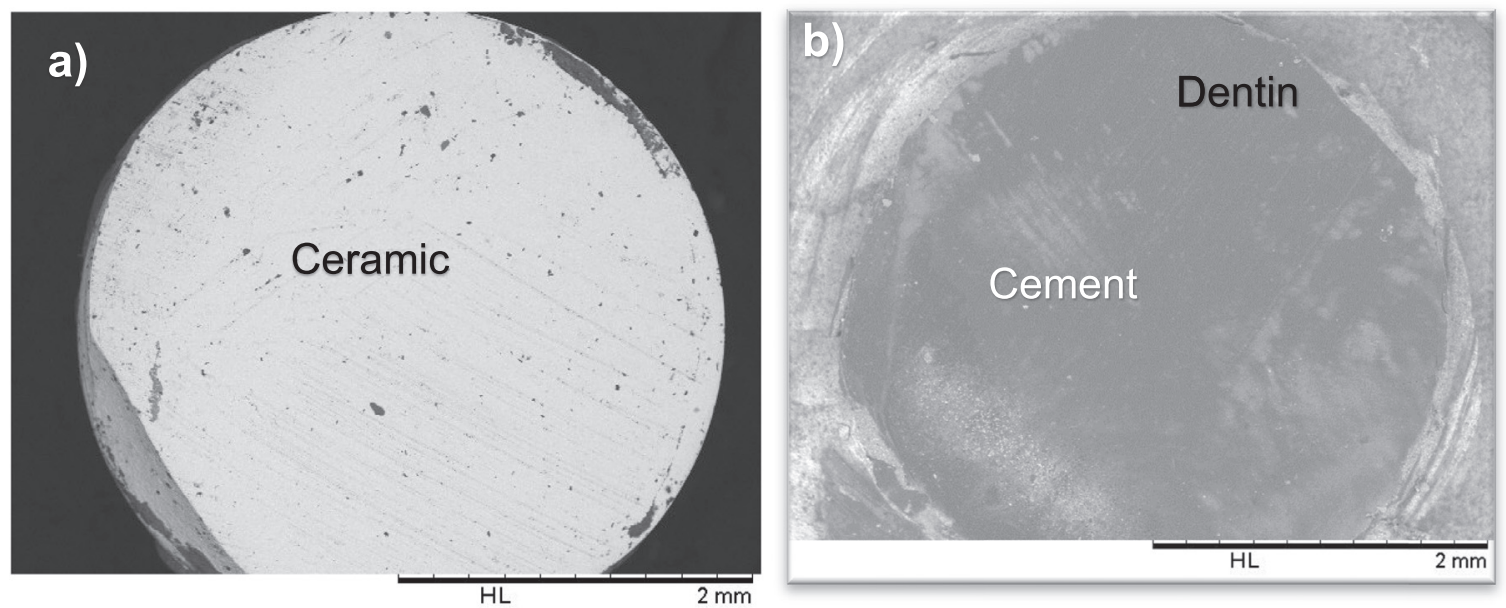

Fig. 4. a-b. Photomicrography (x40) representing adhesive failure of the cement at the a) cement-ceramic (Score A2) and b) cement-dentin interface (Score A1) for the resin cement ULT. 
Table 4

Mean degree of polymerization (DC) and standard deviation (SD) values for the cementation systems and the statistical differences between groups.

\begin{tabular}{lllll}
\hline Groups & $\mathbf{n}$ & Mean DC (\%) & SD & Significant difference \\
\hline PAN & 10 & 49.44 & 20.4 & $\mathrm{~A}$ \\
PAN + ED & 10 & 51.66 & 18.71 & $\mathrm{~A}$ \\
ULT & 10 & 45.8 & 26.69 & $\mathrm{~A}$ \\
ULT + SBU & 10 & 55.19 & 14.23 & $\mathrm{~A}$ \\
MULT & 10 & 68.39 & 11.4 & $\mathrm{~A}$ \\
MULT + P & 10 & 44.79 & 17.13 & $\mathrm{~A}$ \\
\hline
\end{tabular}

cement, dentin) causing stress in the interfaces, along with the water sorption that results in degradation in cement adhesion (Mazzitelli et al., 2008; Pereira et al., 2015). In our study, MULT was the cement system that presented significantly higher values of bond strength both after storage (MULT30D) and after thermocycling (MULT5TC) similar to one previous study (Chen et al., 2016). Although it does not contain MDP in its composition, MULT had a better performance also in another study (Attia et al., 2011), where the authors stated that the use of Monobond $\mathrm{N}$ combined with $\mathrm{Al}_{2} \mathrm{O}_{3}$ air-abrasion or silicatization improves resin cement adhesion to zirconia even after thermal aging since the presence of other phosphate monomers in the primer composition provides a higher chemical bond to ceramic oxides, and consequently higher adhesive strength. On the other hand, the thermocycling may also enhance the post-polymerization of the resin cements and promote a higher bond strength between zirconia and resin cement (Piwowarczyk et al., 2004), which can explain the higher bond strength values after $20.000 \mathrm{TC}$.

Regarding the failure analysis in our study, PAN and MULT showed a greater number of adhesive failures at the cement-dentin interface indicates worse adhesion of these cement systems to dentin. Previous studies also found lower dentin adhesion values with these cement systems where the low results were attributed to partial demineralization of the dentin substrate achieved by both adhesive systems (EDprimer A/B and Primer A/B) (Maciel et al., 1996; Mak et al., 2002). Moreover, contact of the non-polymerized adhesive system with the dual resin cement may promote an adverse chemical interaction, triggering an incomplete polymerization reaction of the adhesive system in the dentin or insufficient time for the acidic monomers to diffuse into the substrate. The presence of catalysts in both the cement and the adhesive system may lead to an acceleration of the polymerization reaction, promoting incomplete polymerization in both the adhesive and cement systems, leading to premature degradation of the interface (Maciel et al., 1996; Aleisa et al., 2013).

Unlike the other two systems, ULT presented a higher number of adhesive failures at the ceramic/cement interface but less adhesive failures to dentin, demonstrating better adhesion to dentin. A greater number of failures in this interface may be due to the presence of many ingredients mixed into the adhesive solution (Faria-e-Silva et al., 2010). As previously reported, a mixture of several constituents in the SBU could prevent adhesion between resin cement-tooth and cement-zirconia interface due to a different reaction in these two substrates (Alves et al., 2016). However, some authors report that although SBU presents a mixture of constituents in the same flask, adhesion at the dentin-cement-zirconia interface is effective (Kim et al., 2015). The presence of MDP, silane and other ingredients in the SBU (i.e. water) is sufficient to firstly allow efficient adhesion with the tooth surface. These adhesives are compatible with the still-wet dentin substrate, where water may act in plasticizing the collapsed collagen network, allowing re-expansion of the spatial interfibrilar spaces and subsequent infiltration of resin monomers and allowing for better SBU/dentin interaction (Inokoshi et al., 2014). Yet, in this study failure types indicated better adhesion with PAN and MULT on zirconia but with ULT on dentin. Nonetheless, none of the investigated primer-cement systems resulted in similar amounts of cohesive cement failures on both dentin and zirconia. Thus, adhesion of the tested systems still remains insufficient to these substrates.

The evaluation of DC was designed in such a way that where clinical procedure of cementing a zirconia restoration was simulated. No significant difference in DC was found between resin cements, even when combined with their correspondingprimer. Several factors may interfere with DC, such as the material composition such as monomers and other components, unintended interactions between adhesive system and cement, photo-polymerization process, and restoration characteristics (i.e. optical properties and thickness) (De Souza et al., 2015). Although higher DC was achieved by these cements in some previous studies (PAN: 78\%; MULT: 61.4\%; ULT: 72\%) (Uhl et al., 2004; Aguiar et al., 2010; Lührs et al., 2014), others have reported that the interaction of these cements with adhesive systems can influence their DC, either by accelerated polymerization reaction due to simultaneous polymerization of the cement and the corresponding primer/adhesive during cementation, increasing the DC, or by the acidity of self-etching adhesive systems that may promote the inactivation of the amine initiator by acid-base reaction, triggering an incomplete polymerization, decreasing the DC (Lührs et al., 2014; Inokoshi et al., 2016).

Nevertheless, the adhesion results obtained in this study along with failure types more in adhesive characteristics are definitely not in the range of adhesion values achieved to resin composites or glassy matrix ceramics (Özcan and Vallittu, 2003; Barbosa et al., 2013). Thus, adhesion to zirconia needs further investigations.

\section{Conclusions}

From this study, the following could be concluded:

1. PAN cement presented the highest number of pre-test failures during aging.

2. Overall when cements with their corresponding primers are compared, the adhesion of zirconia to dentin were less favourable with PAN compared to those of ULT and MULT.

3. Regardless of the cement type, aging through thermocycling significantly decreased the bond strength results when compared water storage while the increased number of cycles above 5.000 up to 10.000 or 20.000 did not significantly affect the results.

4. Failure types indicated better adhesion with PAN and MULT on zirconia but with ULT on dentin.

\section{Acknowledgements}

This study was based on a Master Thesis submitted to Federal University of Rio Grande do Norte (UFRN), Natal, RN, Brazil as part of the requirements for MSci degree.

\section{Clinical relevance}

Quality and durability of adhesion of zirconia to dentin is affected by the primer-cement system based on phosphate monomers. Bond strength results were practically not favourable with all systems and decreased by aging but based on pre-test failures and failure types MULT performed better on zirconia and ULT on dentin.

\section{Conflict of interest}

The authors did not have any commercial interest in any of the materials used in this study.

\section{References}

Aboushelib, M.N., Kleverlaan, C.J., Feilzer, A.J., 2007. Selective infiltration-etching technique for a strong and durable bond of resin cements to zirconia-based materials. J. Prosthet. Dent. 98, 379-388. 
Aguiar, T.R., Di Francescantonio, M., Arrais, C.A., Ambrosano, G.M., Davanzo, C., Giannini, M., 2010. Influence of curing mode and time on degree of conversion of one conventional and two self-adhesive resin cements. Oper. Dent. 35, 295-299.

Aleisa, K., Al-Dwairi, Z.N., Alghabban, R., Goodacre, C.J., 2013. Effect of luting agents on the tensile bond strength of glass fiber posts: an in vitro study. J. Prosthet. Dent. 110, 216-222.

Alves, M.L.L., Campos, F., Bergoli, C.D., Bottino, M.A., Özcan, M., Souza, R.O.A., 2016 Effect of adhesive cementation strategies on the bonding of y-tzp to human dentin. Oper. Dent. 41, 276-283.

Amaral, M., Belli, R., Cesar, P.F., Valandro, L.F., Petschelt, A., Lohbauer, U., 2014. The potential of novel primers and universal adhesives to bond to zirconia. J. Dent. 42, 90-98.

Attia, A., Kern, M., 2011. Long-term resin bonding to zirconia ceramic with a new universal primer. J. Prosthet. Dent. 106, 319-327.

Azimian, F., Klosa, K., Kern, M., 2012. Evaluation of a new universal primer for ceramics and alloys. J. Adhes. Dent. 14, 275-282.

Bachhav, V.C., Aras, M.A., 2011. Zirconia-based fixed partial dentures: a clinical review. Quintessence Int 42, 173-182.

Barbosa, W.F., Aguiar, T.R., Francescantonio, M.D., Cavalcanti, A.N., Oliveira, M.T., Giannini, M., 2013. Effect of water storage on bond strength of self-adhesive resin cements to zirconium oxide ceramic. J. Adhes. Dent. 15, 145-150.

Bottino, M.A., Bergoli, C., Lima, E.G., Marocho, S.M., Souza, R.O., Valandro, L.F., 2014. Bonding of Y-TZP to dentin: effects of Y-TZP surface conditioning, resin cement type, and aging. Oper. Dent. 39, 291-300.

Cavalcanti, A.N., Foxton, R.M., Watson, T.F., Oliveira, M.T., Giannini, M., Marchi, G.M., 2009. Bond strength of resin cements to a zirconia ceramic with different surface treatments. Oper. Dent. 34, 280-287.

Cebe, M.A., Cebe, P.F., Tunçdemir, M.T., Isman, E., 2015. Bonding performance of two newly developed self-adhering materials between zirconium and dentin. Niger. J. Clin. Pract. 18, 221-226.

Chai, J., Chu, F.C.S., Chow, T.W., 2010. Effect of surface treatment on shear bond strength of zirconia to human dentin. J. Prosthodont 20, 173-179.

Chen, C., Chen, Y., Lu, Z., Quian, M., Xie, H., 2017. The effects of water on degradation of the zirconia-resin bond. J. Dent. 64, 23-29.

Dagostin, A., Ferrari, M., 2002. Effect of sealing of dentin on the bond strength of ceramic restorations. Dent. Mater. 18, 304-310.

De Souza, G., Braga, R.R., Cesar, P.F., Lopes, G.C., 2015. Correlation between clinical performance and degree of conversion of resin cements: a literature review. J. Appl. Oral. Sci. 23, 358-368.

Denry, I., Kelly, J.R., 2008. State of the art of zirconia for dental applications. Dent. Mater. 24, 299-307.

Derand, T., Molin, M., Kvam, K., 2005. Bond strength of composite luting cement to zirconia ceramic surfaces. Dent. Mater. 21, 1158-1162.

Egilmez, F., Ergun, G., Cekic-Nagas, I., Vallittu, P.K., Özcan, M., Lassila, L.V.J., 2013. Effect of surface modification on the bond strength between zirconia and resin cement. J. Prosthodont 22, 529-536.

Elsaka, E., 2016. Influence of surface treatment on the bond strength of resin cements to monolithic zirconia. J. Adhes. Dent. 18, 387-395.

Faria-e-Silva, A.L., Fabião, M.M., Arias, V.G., Martins, L.R., 2010. Activation mode effects on the shear bond strength of dual-cured resin cements. Oper. Dent. 35, 515-521.

Inokoshi, M., Poitevin, A., De Munck, J., Minakuchi, S., Van Meerbeek, B., 2014. Bonding effectiveness to different chemically pre-treated dental zirconia. Clin. Oral. Investig. 18, 1803-1812.

Inokoshi, M., Pongprueksa, P., De Munck, J., Zhang, F., Vanmeensel, K., Minakuchi, S., Vleugels, J., Naert, I., Van Meerbeek, B., 2016. Influence of light irradiation through zirconia on the degree of conversion of composite cements. J. Adhes. Dent. 18, $161-171$.

Kim, J.H., Chae, S., Lee, Y., Han, G.H., Cho, B.H., 2014. Comparison of shear test methods for evaluating the bond strength of resin cement to zirconia ceramic. Acta Odontol. Scand. 72, 745-752.

Kim, J.H., Chae, S.Y., Lee, Y., Han, G.J., Cho, B.H., 2015. effects of multipurpose, universal adhesives on resin bonding to zirconia ceramic. Oper. Dent. 40, 55-62.

Lührs, A.K., De Munck, J., Geurtsen, W.3, Van Meerbeek, B., 2014. Composite cements benefit from light-curing. Dent. Mater. 30, 292-301.

Maciel, K.T., Carvalho, R.M., Ringle, R.D., Preston, C.D., Russel, C.M., Pashley, D.H., 1996. The effects of acetone, ethanol, HEMA, and air on the stiffness of human decalcified dentin matrix. J. Dent. Res 75, 1851-1858.

Mak, Y.F.L., Lai, S.C., Cheung, G.S., Chan, A.W., Tay, F.R., Pashley, D.H., 2002. Microtensile bond testing of resin cements to dentin and an indirect resin composite. Dent. Mater. 18, 609-621.

May, L.G., Salvia, A.C.R., Souza, R.O.A., Michida, S.M.A., Valera, M.C., Takahashi, F.E., Bottino, M.A., 2010, effect of sodium ascorbate and the time lapse before cementation after internal bleaching on bond strength between dentin and ceramic. J.
Prosthodont 19, 374-380.

Mazzitelli, C., Monticelli, F., Osorio, R., Casucci, A., Toledano, M., Ferrari, M., 2008. Effect of simulated pulpal pressure on self-adhesive cements bonding to dentin. Dent. Mater. 24, 1156-1163.

Miragaya, L., Maia, L.C., Sabrosa, C.E., De Goes, M.F., Silva, E.M., 2011. Evaluation of self-adhesive resin cement bond strength to yttria-stabilized zirconia ceramic (YTZP) using four surface treatments. J. Adhes. Dent. 13, 473-480.

Munoz, M.A., Luque, I., Hass, V., Reis, A., Loguercio, A.D., Bombarda, N.H., 2013. Immediate bonding properties of universal adhesives to dentine. J. Dent. 41, 404-411.

Nagaoka, N., Yoshihara, K., Feitosa, V.P., Tamada, Y., Irie, M., Yoshida, Y., Van Meerbeek, B., Hayakawa, S., 2017. Chemical interaction mechanism of 10-MDP with zirconia. Sci. Rep. 30, 455-463.

Nakamichi, I., Iwaku, M., Fusayama, T., 1983. Bovine teeth as possible substitute in the adhesion test. J. Dent. Res 62, 1076-1081.

Neiva, G., Yaman, P., Dennisson, J.B., Razzooq, M.E., Lang, B.R., 1998. Resistance to fracture of three all-ceramic systems. J. Esthet. Dent. 10, 60-66.

Ohlmann, B., Rammelsberg, P., Schmitter, M., Schwarz, S., Gabbert, O., 2008. All-ceramic inlay-retained fixed partial dentures: preliminary results from a clinical study. J. Dent. 36, 692-696.

Ortorp, A., Kihl, M.L., Carlsson, G.E., 2012. A 5-year retrospective study of survival of zirconia single crowns fitted in a private clinical setting. J. Dent. 40, 527-530.

Özcan, M., Vallittu, P.K., 2003. Effect of surface conditioning methods on the bond strength of luting cement to ceramics. Dent. Mater. 19, 725-731.

Özcan, M., Bernasconi, M., 2015. Adhesion to zirconia used for dental restorations: a systematic review and meta-analysis. J. Adhes. Dent. 17, 7-26.

Passia, N., Mitsias, M., Lehmann, F., Kern, M., 2016. Bond strength of a new generation of universal bonding systems to zirconia ceramic. J. Mech. Behav. Biomed. Mater. 62, 268-274.

Perdigão, J., Loguercio, A.D., 2014. Universal or multi-mode adhesives: why and how? J. Adhes. Dent. 16, 193-194.

Perdigao, J., Sezinando, A., Monteiro, P.C., 2012. Laboratory bonding ability of a multipurpose dentin adhesive. Am. J. Dent. 25, 153-158.

Pereira, L., Campos, F., Piva, A.M.O.D., Gondim, L.D., Souza, R.O.A., Özcan, M., 2015. Can application of universal primers alone be a substitute for airborne-particle abrasion to improve adhesion of resin cement to zirconia. J. Adhes. Dent. 17, 169-174.

Piwowarczyk, A., Lauer, H.C., Sorensen, J.A., 2004. In vitro shear bond strength of cementing agents to fixed prosthodontic restorative materials. J. Prosthet. Dent. 92, 265-273.

Schley, J.-S., Heussen, N., Reich, S., Fischer, J., Haselhuhn, K., Wolfart, S., 2010. Survival probability of zirconia-based fixed dental prostheses up to $5 \mathrm{yr}$ : a systematic review of the literature. Eur. J. Oral. Sci. 118, 443-450.

Schmitt, J., Holst, S., Wichmann, M., Reich, S., Gollner, M., Hamel, J., 2009. Zirconia posterior fixed partial dentures: a prospective clinical 3-year follow-up. Int J. Prosthodont 22, 597-603.

Soares, F.Z.M., Follak, A., da Rosa, L.S., Montagner, A.F., Lenzi, T.L., Rocha, R.O., 2016. Bovine tooth is a substitute for human tooth on bond strength studies: a systematic review and meta-analysis of in vitro studies. Dent. Mater. 32, 1385-1393.

Solá-Ruíz, M.F., Agustin-Panadero, R., Fons-Font, A., Labaig-Rueda, C., 2015. A prospective evaluation of zirconia anterior partial fixed dental prosthe ses: clinical results after seven years. J. Prosthet. Dent. 113, 578-584.

Souza, R., Barbosa, F., Araújo, G., Miyashita, E., Bottino, M.A., Melo, R., Zhang, Y., 2018. Ultrathin monolithic zirconia veneers: reality or future? Report of a clinical case and one-year follow-up. Oper. Dent. 43, 3-11.

Stober, T., Bermejo, Jl, Rammelsberg, P., Schmitter, M., 2014. Enamel wear caused by monolithic zirconia crowns after 6 months of clinical use. J. Oral. Rehabil. 41, 314-322.

Tanis, M.C., Akaya, C., Karakis, D., 2015. Resin cementation of zirconia ceramics with different bonding agents. Biotechnol. Biotechnol. Equip. 29, 363-367.

Tinschert, J., Natt, G., Mautsch, W., Augthun, M., Spiekermann, H., 2001. Fracture resistance of lithium disilicate-, alumina, and zirconia-based three-unit fixed partial dentures: a laboratory study. Int J. Prosthodont 14, 231-238.

Tsujimoto, A., Barkmeier, W.W., Takamizawa, T., Wilwerding, T.M., Latta, M.A., Miyazaki, M., 2017. interfacial characteristics and bond durability of universal adhesive to various substrates. Oper. Dent. 42, 59-70.

Uhl, A., Michaelis, C., Mills, R.W., Jandt, K.D., 2004. The influence of storage and indenter load on the Knoop hardness of dental composites polymerized with LED and halogen technologies. Dent. Mater. 20, 21-28.

Yang, B., Barloi, A., Kern, M., 2010. Influence of airabrasion on zirconia ceramic bonding using an adhesive composite resin. Dent. Mater. 26, 44-50.

Yun, J., Ha, S., Lee, J., Kim, S., 2010. Effect of sandblasting and various metal primers on the shear bond strength of resin cement to Y-TZP ceramic. Dent. Mater. 26, 650-658. 